\title{
Mucoid Impaction in a 15-Year-Old With Bronchial Atresia
}

\author{
Srinivas Rajagopala MD DM, Uma Devaraj DNB, Smrita Swamy MD, \\ and Anoop Kumar HS MBBS
}

\section{Introduction}

The differential diagnosis of focal lung masses in childhood is large and includes mucoid impaction, congenital masses, inflammatory lung diseases, and neoplastic conditions. Mucoid impaction has a characteristic "finger-inglove" appearance on chest x-ray. The etiology of mucoid impaction can be quickly narrowed by a focused clinical and radiological evaluation. We describe an index patient and discuss the evaluation of mucoid impaction in clinical practice.

\section{Case Summary}

A 15-year-old boy presented with fever and productive cough of 4 weeks duration. Cough was muco-purulent, about $20 \mathrm{~mL} / \mathrm{d}$, without any postural variation. There was no history of hemoptysis, anorexia, or weight loss. He had several episodes of "chest colds" in the past, which usually resolved with oral antibiotics. Each episode of "chest cold" lasted about 10 days, and these episodes occurred once every 2-3 months. The patient did not have any prior admissions for respiratory illness or episodes suggestive of possible aspiration. He denied any abdominal pain, bulky stools, or chronic productive cough. There was no prior history of treated tuberculosis, rhinosinusitis, and exanthematous fevers in childhood. He did not report any important illness in his siblings or family members, and did not smoke or drink alcohol and did not raise pets.

Dr Swamy is affiliated with the Department of Radiodiagnosis; the other authors are affiliated with the Division of Chest Diseases, Department of Medicine, St John's Medical College Hospital, Bengaluru, India.

The authors have disclosed no conflicts of interest.

Correspondence: Srinivas Rajagopala MD DM, Division of Chest Diseases, Department of Medicine, St John's Medical College Hospital, Bengaluru, India 560034. E-mail: visitsrinivasan@gmail.com.

DOI: $10.4187 /$ respcare.01196
Oral levofloxacin was administered for 10 days, with resolution of fever; however, he had a non-resolving radiologic infiltrate 4 weeks after the antibiotic course, and a high-resolution computed tomography (HRCT) of the thorax was performed. CT-thorax showed right middle lobe consolidation, and subsequently the patient underwent fiberoptic bronchoscopy. Bronchoscopy was normal, and bronchoscopic lavage was negative for acid-fast bacilli and sterile by aerobic bacterial cultures. He was referred to this center for further evaluation of the persistent chest infiltrate.

On examination he was afebrile and normotensive, with respiratory rate of 20 breaths $/ \mathrm{min}$ and pulse rate of 100 beats/min. General physical examination was normal (body-mass index of $24 \mathrm{~kg} / \mathrm{m}^{2}$ ). Respiratory examination showed reduced right-mammary breath sounds intensity, with no adventitious sounds. The rest of the physical examination was unremarkable. Sputum was negative for acid-fast bacilli on 2 occasions by Ziehl-Neelsen staining, and sterile by aerobic bacterial cultures. Review of the chest X-ray (Fig. 1) showed classic "finger-in glove" appearance in the right lung (black arrow), with evidence of increased lucency focally (see Fig. 1, white arrow). Sputum culture was negative for Aspergillus fumigatus, and serum total immunoglobulin E levels were normal $(<30 \mathrm{IU} /$ $\mathrm{mL})$. Spirometry was normal: $\mathrm{FEV}_{1} / \mathrm{FVC} 78 \%, \mathrm{FEV}_{1} 2.68 \mathrm{~L}$ (88\% predicted), FVC 3.43 L (84\% predicted). Review of the HRCT-thorax showed a branching non-enhancing opacity of +10 Hounsfield units attenuation; there was evidence of focal increased attenuation in the lateral segment of the right middle lobe (Fig. 2, left, and Fig. 3). The lateral segmental bronchus of the middle lobe was also absent.

A diagnosis of bronchial atresia was made, and the patient was offered surgery because of recurrent symptoms. However, he declined the same and remains asymptomatic, with no symptom recurrence at 6 months of follow-up.

\section{Discussion}

Bronchial atresia is a congenital abnormality resulting from intra-uterine focal interruption of a segmental or sub- 


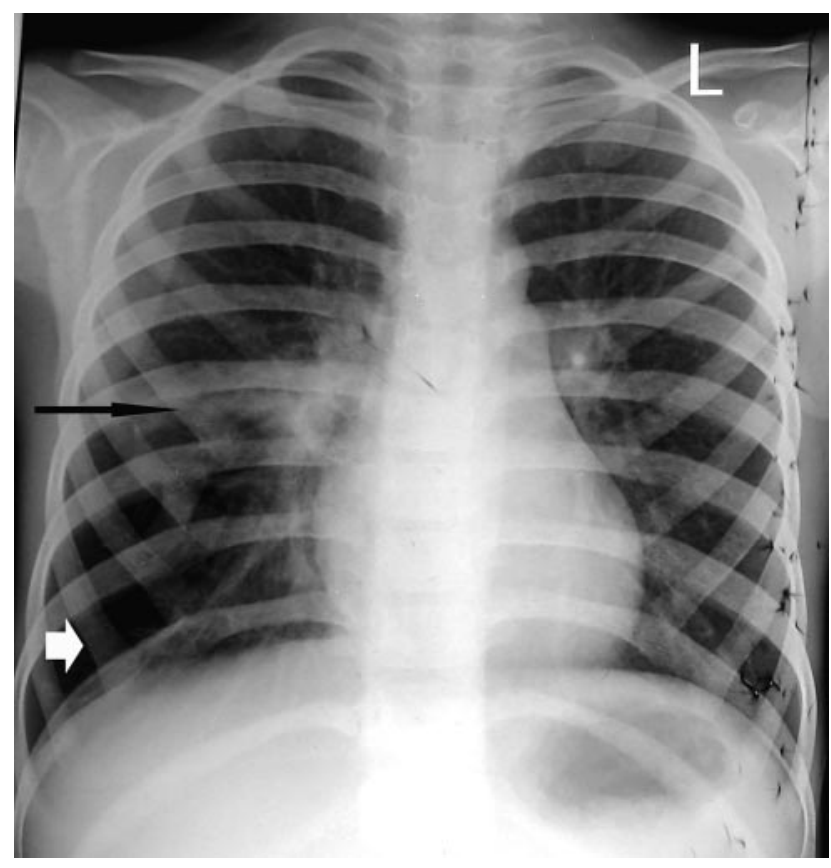

Fig. 1. Chest x-ray showing "finger-in glove" appearance in the right lung (black arrow), suggesting mucus impaction, with evidence of increased lucency focally (white arrow).

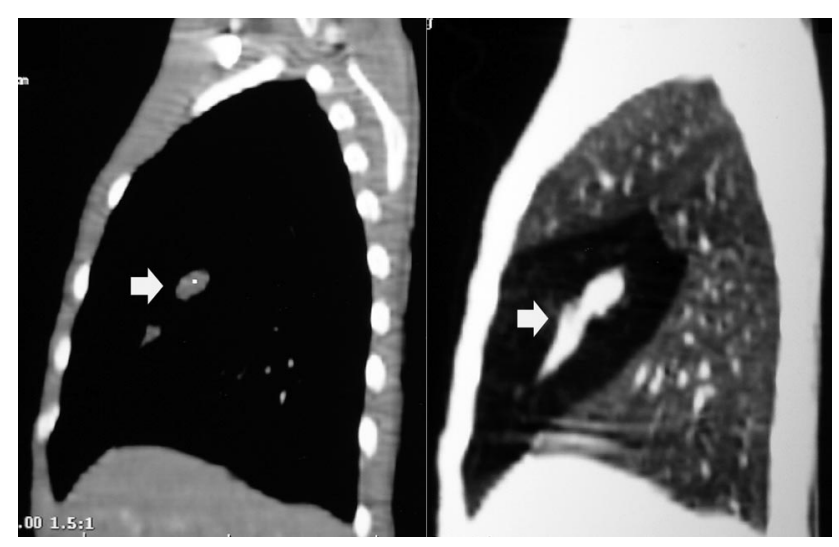

Fig. 2. Composite image of the computed tomography of the thorax (right, mediastinal window) shows a branching, non-enhancing tubular opacity of +10 Hounsfield units attenuation, with evidence of distal hyperinflation in the lateral segment of the right middle lobe. Lung windows (left) show that the lateral sub-segmental bronchus is absent, and this is associated with sub-segmental hyperinflation. No evidence of bronchiectasis, centrilobular nodules, or high attenuation mucus is seen.

segmental bronchus due to ischemia. ${ }^{1}$ The exact etiology of bronchial atresia is unknown. The timing of intra-uterine insult may be associated with the site of bronchial atresia, as the lobar bronchi, sub-segmental bronchi, and distal bronchioles appear by the 5th, 6th, and 16th weeks of fetal development respectively. ${ }^{2}$ The airways distal to the atresia are normal, and are supplied by collateral ventilation through intra-alveolar pores of Kohn, bronchoal-

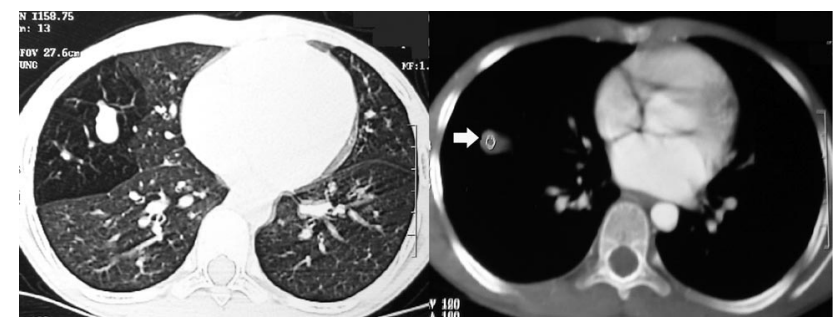

Fig. 3. Composite image of the sagittal reconstruction of the computed tomography images, with mediastinal windows (left) showing the central branching, non-enhancing tubular opacity of +10 Hounsfield units attenuation, due to mucus plugging, and the lung windows (right) showing the sub-segmental distal hyperinflation.

Table 1. Differential Diagnosis of Mucoid Impaction

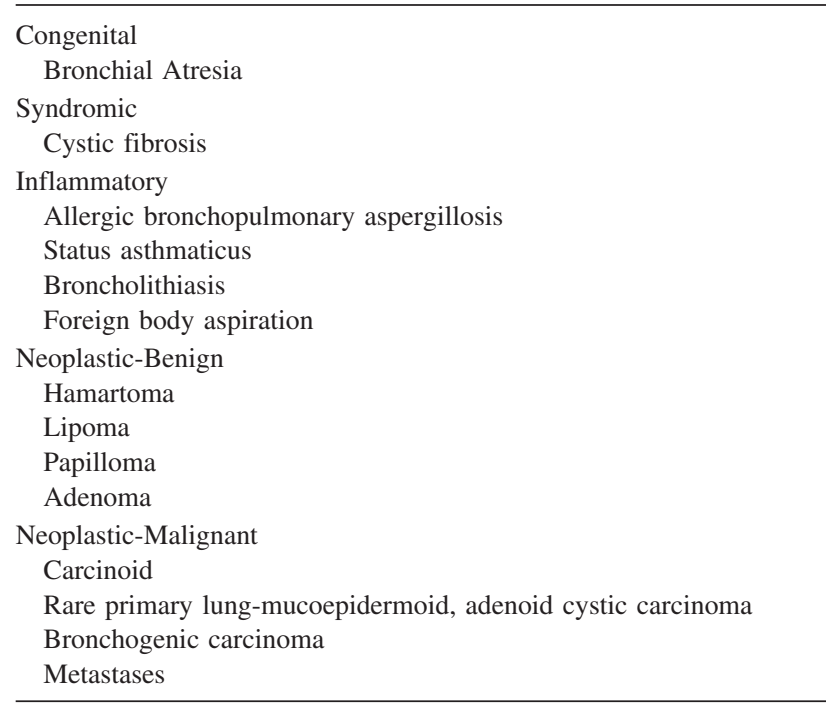

veolar channels of Lambert, and inter-bronchiolar channels. The most common location is the apico-posterior segment of the left upper lobe. ${ }^{3}$

Radiologically, bronchial atresia is associated with a triad of findings: focal interruption of a bronchus; associated distal mucus impaction (bronchocele) that is visible as a branching tubular or ovoid area of increased attenuation; and associated hyperinflation of the obstructed pulmonary segment. ${ }^{4}$ The central atretic bronchus leads to mucus impaction, and this is associated with distal hyperinflation due to collateral ventilation. Mucoid impaction in the central airways may be visible as by tubular or branching opacities that resemble fingers and this is described as "finger-in-glove sign" (see Fig. 1). Atypical appearances with ovoid appearances may occur with distal impaction or impaction in non-dilated central airways.

The hyperlucent lung surrounding the bronchocele represents a combination of air trapping and focal parenchymal oligemia. Oligemia in mucoid impaction is secondary 
to intrapulmonary vascular compression and hypoxic vasoconstriction. ${ }^{5}$ If hyperinflation is not evident, expiratory images can accentuate this finding. When the triad of focal interruption of a bronchus, bronchocele, and associated hyperinflation of the obstructed pulmonary segment are present on CT, a confident clinical diagnosis of bronchial atresia can be made. ${ }^{5}$

The differential diagnosis of mucoid impaction is large (Table 1), but can be narrowed by a focused history and examination. ${ }^{6}$ Bronchial atresia, allergic bronchopulmonary aspergillosis, and cystic fibrosis are the 3 most common conditions associated with mucoid impaction.

Bronchial atresia is usually benign and incidentally detected. The mean age at diagnosis is 17 years. A 2:1 male preponderance is seen. If symptomatic, recurrent pulmonary infections, mild wheezing, and dyspnea are the most common presenting symptoms. The differential diagnosis includes the other causes of mucus impaction (see Table 1) and pulmonary arteriovenous malformation. Contrast-CT can confidently exclude the latter. ${ }^{6}$ Complications include recurrent infections or pneumothoraces. Surgery is usually reserved for symptomatic individuals. When surgery is considered, parenchyma-sparing segmentectomy (or lobectomy) should be considered. ${ }^{5}$

\section{Teaching Points}

- Mucus impaction in central airways leads to finger-inglove appearance.

- The differential diagnosis of mucoid impaction can be narrowed by a focused history and examination.

- The triad of focal interruption of a bronchus, bronchocele, and associated hyperinflation of the obstructed pulmonary segment leads to a confident diagnosis of bronchial atresia on CT.

\section{REFERENCES}

1. Ramsay BH. Mucocele of the lung due to congenital obstruction of a segmental bronchus; a case report; relationship to congenital cystic disease of the lung and to congenital bronchiectasis. Dis Chest 1953; 24(1):96-103.

2. Kinsella D, Sissons G, Williams MP. The radiological imaging of bronchial atresia. Br J Radiol 1992;65(776):681-685.

3. Meng RL, Jensik RJ, Faber LP, Matthew GR, Kittle CF. Bronchial atresia. Ann Thorac Surg 1978;25(3):184-192.

4. Cohen AM, Solomon EH, Alfidi RJ. Computed tomography in bronchial atresia. AJR Am J Roentgenol 1980;135(5):1097-1099.

5. Gipson MG, Cummings KW, Hurth KM. Bronchial atresia. Radiographics 2009;29(5):1531-1535.

6. Martinez S, Heyneman LE, McAdams HP, Rossi SE, Restrepo CS, Eraso A. Mucoid impactions: finger-in-glove sign and other CT and radiographic features. Radiographics 2008;28(5):1369-1382. 